\title{
Third generation SUSY searches in CMS
}

\author{
Eric Chabert* on behalf the CMS Collaboration \\ IPHC, IN2P3-CNRS, Strasbourg University \\ E-mail: Eric.Chabertecern.ch
}

This conference report presents searches for direct pair production of third generation squark performed with a dataset of proton-proton collision at $\sqrt{s}=13 \mathrm{TeV}$ collected by the CMS experiment at the LHC, The data used correspond to an integrated luminosity of $2.3 \mathrm{fb}^{-1}$. No deviation from the standard model background is observed. The results are interpreted in the context of few simplified models. Within such models, stop masses up to $780 \mathrm{GeV}$ and sbottom masses up to $890 \mathrm{GeV}$ have been excluded at 95\% CL for low mass neutralino.

Fourth Annual Large Hadron Collider Physics

13-18 June 2016

Lund, Sweden

${ }^{*}$ Speaker. 


\section{Introduction}

The standard model (SM) has been extremely successful at describing particle physics phenomena since decades. However, it suffers from shortcomings, such as a mechanism to stabilize the Higgs boson mass against radiative corrections referred as the hierarchy problem and an inability to provide a dark matter candidate. Supersymmetry (SUSY) is an extension to the SM, which introduce a new symmetry relating fermions and bosons. For every known boson (fermion) of the $\mathrm{SM}$, it postulates the existence of a yet unseen fermionic (bosonic) partner. The contributions of the top quark supersymmetric partner, also called stop, to the Higgs boson mass would partially compensates the top quark ones solving thus the hierarchy problem. The argument of naturality suggests that the stop mass should be within the $\mathrm{TeV}$ range and thus accessible at the LHC. Under the assumption that R-parity is conserved, a dark matter candidate is also provided in the form of the lightest supersymmetric particle (LSP). In many models, the LSP is the lightest neutralino, $\tilde{\chi}_{1}^{0}$.

This note presents results of several searches for third generation squark pair production using a dataset corresponding to an integrated luminosity of $2.3 \mathrm{fb}^{-1}$ of proton-proton (pp) collisions collected at a center-of-mass energy of $13 \mathrm{TeV}$ with the CMS detector [1] at LHC in 2015. The results of similar searches were previously reported by the ATLAS and CMS collaborations using datasets of 7 and $8 \mathrm{TeV}$ pp collisions [2, 3, 4, 5, 6, 7, 8, 9, 10, 11] and earlier by CDF and D0 collaborations at the Tevatron. No excess above the SM expectations was observed and the results of those searches were used to place lower limits on the mass of the squarks for different decay scenarios. Depending on the decay mode, these searches probed mass up to about $700 \mathrm{GeV}$ for both stop and sbottom (supersymmetric partner of the bottom quark).

The presented searches have been interpreted in the context of simplified model spectra where the possible decays considered for the lightest stop $\tilde{t_{1}}$ or sbottom $\tilde{b_{1}}$ are: $\tilde{t_{1}} \rightarrow t \tilde{\chi_{1}^{0}}, \tilde{t_{1}} \rightarrow b \tilde{\chi_{1}^{ \pm}}$, $\tilde{t_{1}} \rightarrow c \tilde{\chi_{1}^{0}}$ and $\tilde{b_{1}} \rightarrow b \tilde{\chi_{1}^{0}}$. In the following, when the existence of a low mass chargino $\tilde{\chi_{1}^{ \pm}}$is postulated, it assumes that it is nearly mass degenerate with the $\tilde{\chi}_{1}^{0}(\Delta m=5 \mathrm{GeV})$. Considering a pair of squarks, several decay chain are possible depending if the asymetric decays are considered or not. The only asymetric case treated in that note is the process: $\tilde{t_{1}} \tilde{t_{1}} \rightarrow b \tilde{\chi_{1}^{ \pm}} t \tilde{\chi_{1}^{0}}$. The Feynman diagram corresponding to the processes studied in that note are shown in Fig. 1.

The next section will be dedicated to the stop pair production either in final states with zero [12] or one lepton[13]. Those analyses treat several decays chains including an intermediate $\tilde{\chi_{1}^{ \pm}}$. Dedicated strategies have been developed to cover a large spectrum of kinematics related to the mass spectra, from the compressed regime where particles are almost degenerated up to boosted regime where the mass difference $(\Delta m)$ is large. The last section is dedicated to a search for sbottom pair production [14] where the results where also reinterpreted for $\tilde{t_{1}} \rightarrow c \tilde{\chi_{1}^{ \pm}}$as charm quarks can be tagged by the b-tagging algorithm.

The analyses use CMS standard reconstruction algorithms based on particle flow. For lepton selection, the isolation criteria is based on a cone size dependent on lepton $p_{\mathrm{T}}$ in order to keep good performances over a large $p_{\mathrm{T}}$ spectrum. In order to enhance the observability of the signal, the analyses described below use appropriate event topology or event hypothesis variables that will be introduce later. More technical details can be found in the public analysis summaries [12][13][14]. 


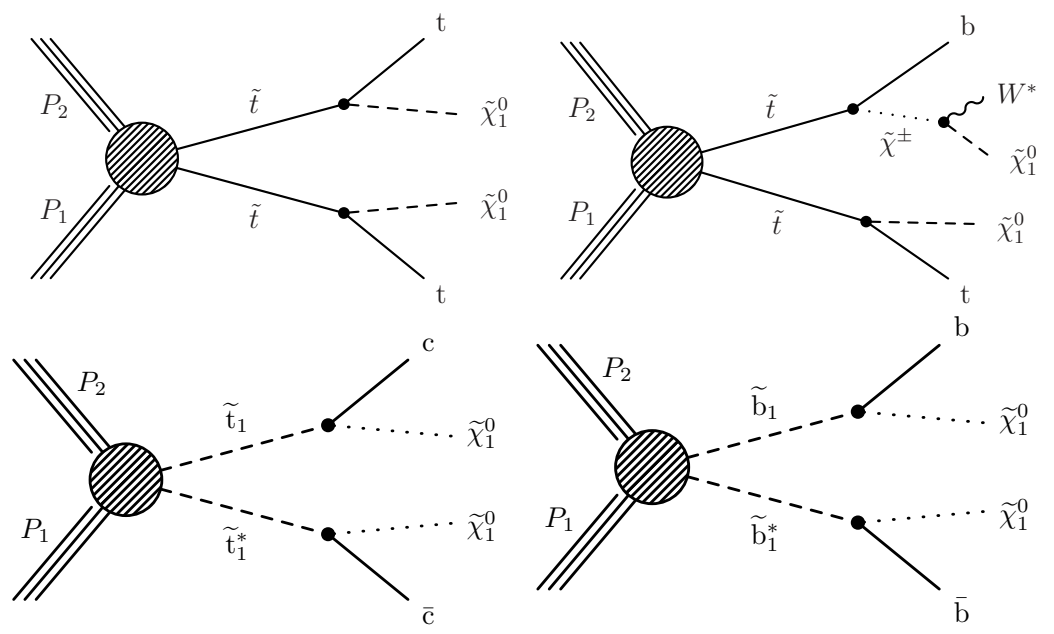

Figure 1: Feynman diagrams showing the pair production of top or bottom squarks followed by their decays according to $\tilde{t_{1}} \rightarrow b \tilde{\chi_{1}^{0}}$ (top left), $\tilde{t_{1}} \rightarrow b \tilde{\chi_{1}^{ \pm}}$(top right, h asymmetric decay displayed), $\tilde{t}_{1} \rightarrow c \tilde{\chi_{1}^{0}}$ (bottom left) and $\tilde{b_{1}} \rightarrow b \tilde{\chi_{1}^{0}}$ (bottom right).

\section{Direct stop pair production searches}

\subsection{Fully hadronic channel}

This section present two independent searches in the fully hadronic channel [12] that have been performed, targeting different corners of the mass spectrum phase space. In both cases, the event selection is based on a veto on identified leptons, large $E_{\mathrm{T}}^{\text {miss }}$, multiple jets and at least one jet identified as originating from the hadronization of a $b$ quark. One of the dominant sources of the $\mathrm{SM}$ background originates from $\mathrm{t} \overline{\mathrm{t}}$ or $\mathrm{W}$ boson production in association with jets in events with leptonic $\mathrm{W}$ boson decays. Events in which a $\mathrm{Z}$ boson, produced in association with jets, decays to neutrinos also provide a significant contribution to the SM background. They rely on the use of top quark tagging algorithms and the categorization of events into exclusive search regions based on kinematic observables related to jets and the missing transverse energy $E_{\mathrm{T}}^{\text {miss }}$.

\section{“High purity top tagging” (HPTT) analysis}

This analysis utilizes a high purity top tagging algorithm to identify top quarks. The HPTT analysis is designed to have improved sensitivity for mixed decay models, and compressed models $\tilde{t_{1}} \tilde{t_{1}} \rightarrow t \tilde{\chi_{1}^{0}} t \tilde{\chi_{1}^{0}}$ with a small mass difference $(\Delta m)$ between the $\tilde{t_{1}}$ and $\tilde{\chi_{1}^{0}}$, including models in which the $\tilde{t_{1}}$ decays via an off-shell top quark.

The event selection requires no isolated leptons (electron, muon or hadronic tau), at least five jets which at least two (one) are tagged by b-tagging algorithm using a loose (medium) operating point. Additionaly to reduce QCD multijet events where $E_{\mathrm{T}}^{\text {miss }}$ originates from $p_{\mathrm{T}}$ mismeasurement, a cut on the angle between $E_{\mathrm{T}}^{\text {miss }}$ and the jets defined as followed is used:

$$
\operatorname{Min}\left[\left|\Delta \phi\left(E_{\mathrm{T}}^{\mathrm{miss}}, \mathrm{j}_{1}\right)\right|,\left|\Delta \phi\left(E_{\mathrm{T}}^{\mathrm{miss}}, \mathrm{j}_{2}\right)\right|,\left|\Delta \phi\left(E_{\mathrm{T}}^{\mathrm{miss}}, \mathrm{j}_{3}\right)\right|,\left|\Delta \phi\left(E_{\mathrm{T}}^{\mathrm{miss}}, \mathrm{j}_{4}\right)\right|\right] \equiv \Delta \phi_{1234}>0.5
$$


, where $\mathrm{j}_{1}, \mathrm{j}_{2}, \mathrm{j}_{3}, \mathrm{j}_{4}$ are the four leading jets in $p_{\mathrm{T}}$. One of the main background comes from t⿱t events where a lepton from $\mathrm{W}$ decay is not reconstructed or identified and referred later as lost lepton. In such events, the transverse mass of $E_{\mathrm{T}}^{\text {miss }}$ and the b quark from the same top decay as the lost lepton has a kinematic end point at the mass of the top quark.

The top tagging algorithm used for the HPTT analysis identifies top quark decays with high purity, at the cost of lower signal efficiency. Top quark candidates are identified by the "CMS top tagging" (CTT) algorithm, which makes use of jet substructure and jet mass observables in order to identify top quark decays. It is based on fat jet reconstructed with the anti- $k_{\mathrm{T}}$ algorithm with a distance parameter of 0.8 . Only jets with $p_{\mathrm{T}}>400 \mathrm{GeV}$ and within the tracker acceptance are retained. Then the jets are decomposed into at least three subjets, where the invariant mass of these subjets is also required to be consistent with the top quark mass $(140-250 \mathrm{GeV})$. The minimum invariant mass of any pair of the three highest $p_{\mathrm{T}}$ subjets must exceed $50 \mathrm{GeV}$, in order to be consistent with the hadronic decay of a W boson.

The observable $M_{\mathrm{T}}\left(\mathrm{b}_{1,2}, E_{\mathrm{T}}^{\text {miss }}\right)$, is defined as

$$
M_{\mathrm{T}}\left(\mathrm{b}_{1,2}, E_{\mathrm{T}}^{\mathrm{miss}}\right) \equiv \operatorname{Min}\left[M_{\mathrm{T}}\left(\mathrm{b}_{1}, E_{\mathrm{T}}^{\mathrm{miss}}\right), M_{\mathrm{T}}\left(\mathrm{b}_{1}, E_{\mathrm{T}}^{\mathrm{miss}}\right)\right],
$$

where $b_{1}, b_{2}$ are the two selected $b$-tagged jets with the highest b-tagging discriminator. In order to benefit from the separation power provided by this variable, but to not loose signal efficiency for low $\Delta m$ mass spectra scenario, this variable is used for categorization.

Finally, the events are categorized into 50 exclusive signal regions based on $M_{\mathrm{T}}\left(\mathrm{b}_{1,2}, E_{\mathrm{T}}^{\text {miss }}\right)$, the number of jets $N_{\text {jets }}$, the number of b-tagged jets $N_{\mathrm{b}-\text { tags }}$, the number of top-tagged jets $N_{\text {top }}$ and the value of $E_{\mathrm{T}}^{\mathrm{miss}}$.

\section{'High efficiency top tagging”' (HETT) analysis}

This analysis utilizes a custom, highly efficient algorithm to tag top-like objects in events and uses their kinematic properties as input to the computation of the "stransverse" mass $\left(M_{\mathrm{T} 2}\right)$ variable. The HETT analysis is largely oriented towards models where $\tilde{\tau_{1}} \tilde{t_{1}} \rightarrow t \tilde{\chi_{1}^{0}} t \tilde{\chi_{1}^{0}}$ with medium to large $\Delta m$ between the $\tilde{t_{1}}$ and $\tilde{\chi_{1}^{0}}$.

The event selection requires at least 4 jets with the leading two jets required to have an transverse momenta $p_{\mathrm{T}}>50 \mathrm{GeV}$. The scalar sum of jet $p_{\mathrm{T}}$ 's must satisfy $H_{\mathrm{T}} \geq 500 \mathrm{GeV}$, A requirement on the angle between $E_{\mathrm{T}}^{\text {miss }}$ and the first three leading jets, $\Delta \phi\left(E_{\mathrm{T}}^{\text {miss }}, j_{1,2,3}\right)>0.5,0.5,0.3$, is applied to remove events from QCD multijet processes. Finally, requirements that $N_{\text {top }} \geq 1, N_{\mathrm{b}-\text { tags }} \geq 1$, and $M_{\mathrm{T} 2}>200 \mathrm{GeV}$ are applied.

The top-tagging algorithm starts with jets reconstructed using the anti- $k_{\mathrm{T}}$ algorithm with distance parameter $R=0.4$, and tests various combinations of three jets within a large cone of radius 1.5 in $\eta-\phi$ space in order to reconstruct hadronically-decaying top quarks from resolved jets. This approach ensures high top-tagging efficiency at $p_{\mathrm{T}}$ below the range targeted by the boosted top quark algorithms. The efficiency of the algorithm is further improved at high $p_{\mathrm{T}}$ by using the jet mass to identify boosted scenarios in which decay products from the $\mathrm{W}$ boson or top quark are merged into a single jet.

Finally, the events are categorized into 37 exclusive signal regions based on $M_{\mathrm{T} 2}, N_{\mathrm{b}-\mathrm{tags}}, N_{\mathrm{top}}$ and $E_{\mathrm{T}}^{\mathrm{miss}}$. 


\section{Results and interpretation}

The data are consistent with the background expected from the SM processes further the results are interpreted in term of limits for simplified models. The Figure 2 shows the 95\% CL exclusion limits obtained by the HPTT and HETT analyses, respectively, for simplified models where stop exclusively decays as $\tilde{t_{1}} \rightarrow t \tilde{\chi_{1}^{0}}$, and in the mixed scenario assuming a $50 \%$ branching fraction for each of the two decay modes $\left(\tilde{t_{1}} \rightarrow t \tilde{\chi_{1}^{0}} / \tilde{t_{1}} \rightarrow b \tilde{\chi}_{1}^{ \pm}\right)$. In the latter case, the $\tilde{\chi_{1}^{ \pm}}$and $\tilde{\chi_{1}^{0}}$ are assumed to be nearly degenerate in mass, with a $5 \mathrm{GeV}$ difference between their masses. The limits obtained by the two analyses are comparable. Using the $2.3 \mathrm{fb}^{-1}$ dataset, stop masses up to $780 \mathrm{GeV}$ and chargino masses up to $260 \mathrm{GeV}$ are probed in the first scenario. In the mixed decay scenario, stop masses up to $750 \mathrm{GeV}$ and neutralino masses up to $280 \mathrm{GeV}$ are probed.

\subsection{Leptonic channel}

This section presents a search for direct stop pair production based on the presence of an isolated lepton which target signal events where only one of $\mathrm{W}$ boson decay leptonically. The event selection requires exactly one identified and isolated electron or muon, at least two jets where at least one of them being b-tagged and $E_{\mathrm{T}}^{\text {miss }}>250 \mathrm{GeV}$. To suppress single-lepton backgrounds originating from semi-leptonic $\mathrm{t} \overline{\mathrm{t}}, \mathrm{W}+$ jets, and single top processes, a requirement on the transverse mass of the lepton-neutrino system is imposed. It is defined as:

$$
M_{\mathrm{T}}=\sqrt{2 p_{\mathrm{T}}^{l} E_{\mathrm{T}}^{\mathrm{miss}}(1-\cos (\phi))}
$$

where $\phi$ is the angle between the transverse momentum of the lepton and $E_{\mathrm{T}}^{\text {miss }}$. Background processes containing a single leptonically-decaying $W$ boson have a kinematic endpoint $M_{T}<M_{W}$, modulo effects of the detector resolution and off-shell $W$ mass effects. The requirement on $M_{T}>$ $150 \mathrm{GeV}$ significantly reduces single-lepton backgrounds. To further reduce the $\overline{\mathrm{t}}$ background, a requirement on the azimuthal angle between $\vec{p}_{\mathrm{T}}^{\text {miss }}$ and the closest of the two leading $E_{T}$ jets $(\Delta \phi)$ to be larger than 0.8 radians is imposed. The remaining background after these selections is dominated by dilepton events from $\mathrm{t} \overline{\mathrm{t}}$ and $\mathrm{tW}$ production, where one of the leptons is not reconstructed or identified (lost lepton). In order to reduce this background, a veto on a second lepton is applied with looser isolation and $p_{\mathrm{T}}$ criteria as well as a veto on a isolated hadronic tau lepton and a veto on an isolated track compatible with the primary vertex.

The baseline search strategy rely on the presence of at least four jets corresponding to the four quarks expected for the top pair semi-leptonic decay. Events are then categorized based on the value of the $M_{\mathrm{T} 2}^{\mathrm{W}}$ variable:

$$
\begin{aligned}
M_{\mathrm{T} 2}^{\mathrm{W}}=\min \left\{m_{y},\right. \text { consistent with: } & {\left[p_{1}^{2}=0,\left(p_{1}+p_{\ell}\right)^{2}=p_{2}^{2}=M_{W}, \vec{p}_{\mathrm{T}}^{1}+\vec{p}_{\mathrm{T}}^{2}=\vec{E}_{\mathrm{T}}^{\mathrm{miss}},\right.} \\
& \left.\left.\left(p_{1}+p_{\ell}+p_{b_{1}}\right)^{2}=\left(p_{2}+p_{b_{2}}\right)^{2}=m_{y}^{2}\right]\right\},
\end{aligned}
$$

where $m_{y}$ is the fitted mother particle mass, and $p_{1}, p_{2}, b_{1}$, and $b_{2}$, are the decay components. It attempts to reconstruct the events under the assumption that it has originated in a $t \bar{t} \rightarrow \ell \ell$ process with one undetected lepton and thus helps to discriminate signal from the dominant dilepton $t \bar{t}$ background. For large mass differences $(\Delta M)$ between the top squark and the neutralino, the $M_{\mathrm{T} 2}^{\mathrm{W}}>$ $200 \mathrm{GeV}$ requirement significantly reduces the background while maintaining reasonable signal 

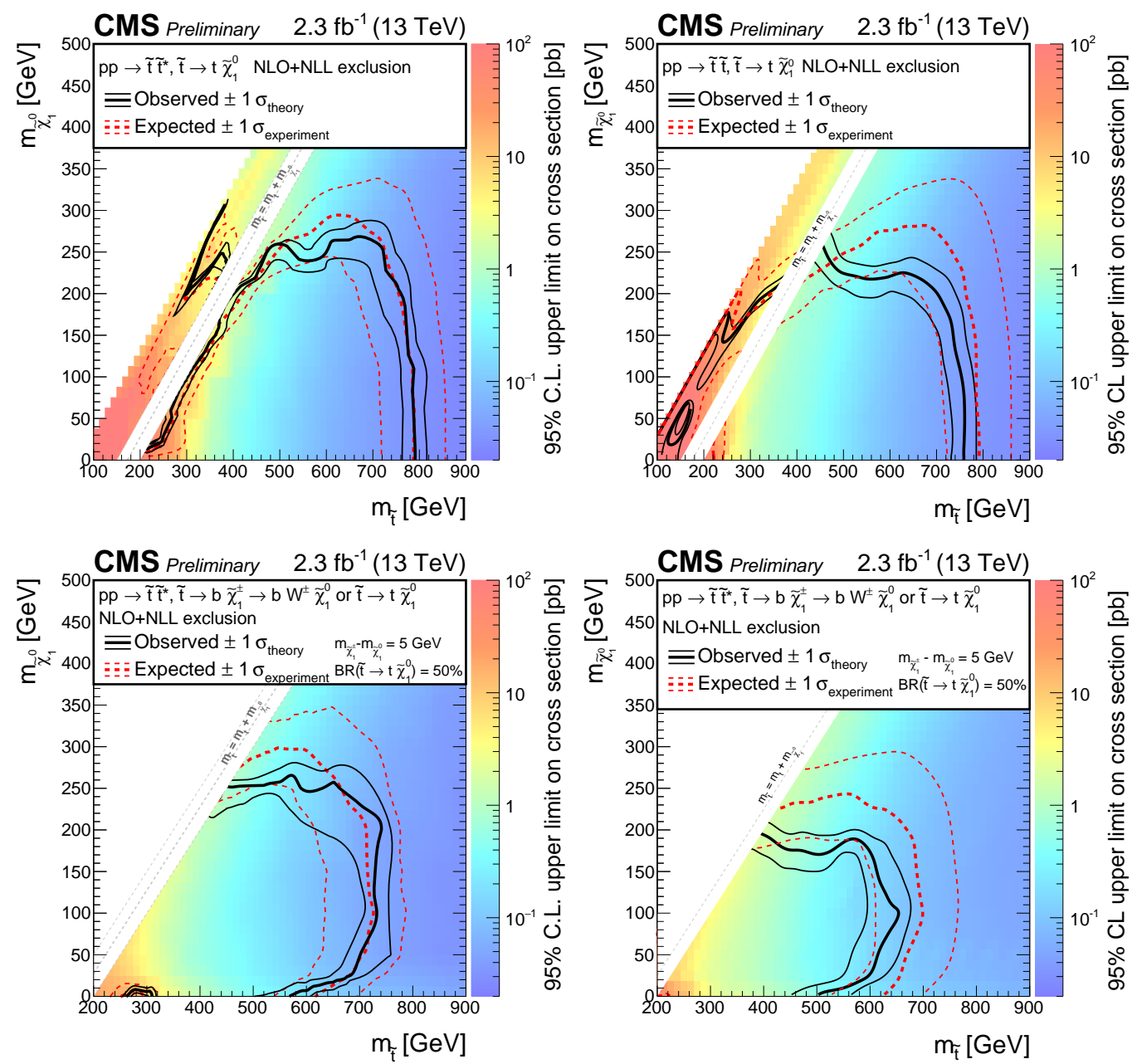

Figure 2: HPTT (left) and HETT (right) exclusion limits at 95\% CL for simplified models of top squark pair production. The results on the top figures correspond to the case where the stop exclusively decay into $\tilde{t_{1}} \rightarrow t \tilde{\chi_{1}^{0}}$. The white diagonal band corresponds to $\left|m_{\tilde{t}_{1}}-m_{t}\right|<25 \mathrm{GeV}$. The results on the bottom figures correspond to a model where a $50 \%$ branching ratio for each of the $\tilde{t_{1}} \rightarrow t \tilde{\chi_{1}^{0}} / \tilde{t_{1}} \rightarrow b \tilde{\chi_{1}^{ \pm}}$modes and a $5 \mathrm{GeV}$ mass difference between the $\tilde{\chi}_{1}^{ \pm}$and $\tilde{\chi_{1}^{0}}$ (right). The solid black curves represent the observed exclusion contours with respect to NLO+NLL cross section calculations.and the corresponding \pm 1 standard deviations. The dashed red curves indicate the expected exclusion contour and the \pm 1 standard deviations with experimental uncertainties.

efficiency. To preserve sensitivity to both high and low $\Delta M$ scenarios, this variable is used for categorization.

In signal scenarios with a large $\Delta M$, a significant fraction of events can contain two quarks that merge into a single jet due to the large boost of the hadronically decaying top quark or $\mathrm{W}$ boson. These events would fail the four-jet requirement. To recover acceptance for such a topology, additional search regions with 3 jets and $M_{\mathrm{T} 2}^{\mathrm{W}}>200 \mathrm{GeV}$ are considered.

To increase the sensitivity of this analysis to a mixed decay scenario corresponding to the process $\tilde{t_{1}} \tilde{t_{1}} \rightarrow t \tilde{\chi_{1}^{0}} b \tilde{\chi_{1}^{ \pm}}$with nearly degenerate chargino and neutrino, search regions with exactly 
two jets are added. In events with low jet multiplicity, a modified version of the topness variable provides improved dilepton $\overline{\mathrm{t}}$ rejection,

$$
t_{\mathrm{mod}}=\ln (\min S) \text { with } S\left(\vec{p}_{W}, p_{v, z}\right)=\frac{\left(m_{W}^{2}-\left(p_{v}+p_{\ell}\right)^{2}\right)^{2}}{a_{W}^{4}}+\frac{\left(m_{t}^{2}-\left(p_{b}+p_{W}\right)^{2}\right)^{2}}{a_{t}^{4}} .
$$

This variable improve the rejection of lost lepton background more than $M_{\mathrm{T} 2}^{\mathrm{W}}$ for low jet multiplicity. Finally, events are categorized into nine exclusive signal regions based on the jet multiplicity, $M_{\mathrm{T} 2}^{\mathrm{W}}$ and $E_{\mathrm{T}}^{\text {miss }}$.

\section{Results and interpretation}

The data are consistent with the background expected from the SM processes further the results are interpreted in term of limits for simplified models. Fig 3 shows the 95\% CL exclusion limits obtained for a simplified model where the stop exclusively decays into $\tilde{t_{1}} \rightarrow t \tilde{\chi_{1}^{0}}$. Using the $2.3 \mathrm{fb}^{-1}$ dataset, top squark masses are excluded up to $730 \mathrm{GeV}$ for a massless $\tilde{\chi}_{1}^{0}$ and $\tilde{\chi}_{1}^{0}$ masses up to $220 \mathrm{GeV}$ for a $500 \mathrm{GeV}$ top squark mass. The results are also interpreted in the context of models of top squark pair production considering two different decay modes for the top squark: $\tilde{t_{1}} \rightarrow t \tilde{\chi_{1}^{0}}$, or $\tilde{t_{1}} \rightarrow b \tilde{\chi_{1}^{ \pm}}$where the chargino is nearly mass-degenerate with the neutralino. Figure 4 shows the expected (left) and observed (right) $95 \%$ CL exclusion limits for top quark pair production with different branching fractions for the $\tilde{t_{1}} \rightarrow t^{(*)}{\tilde{\chi_{1}^{0}}}_{\text {and }} \tilde{t}_{1} \rightarrow b \tilde{\chi_{1}^{0}}$ decays. We only consider the case where the chargino is nearly mass-degenerate with the neutralino. The exclusion limits worsen with increasing $\tilde{t_{1}} \rightarrow b \tilde{\chi_{1}^{0}}$ branching fraction because the current analysis has no sensitivity to the $b \chi_{1}^{+} \bar{b} \chi_{1}^{-}$diagram and it has less sensivitiy to the $t^{(*)} \chi_{1}^{0} b \chi_{1}^{+}$diagram than the $t^{(*)} \chi_{1}^{0} \bar{t}^{(*)} \chi_{1}^{0}$ diagram because a lepton from the chargino decay is too soft to pass the selection requirements due to the small splitting between the chargino and the neutralino.

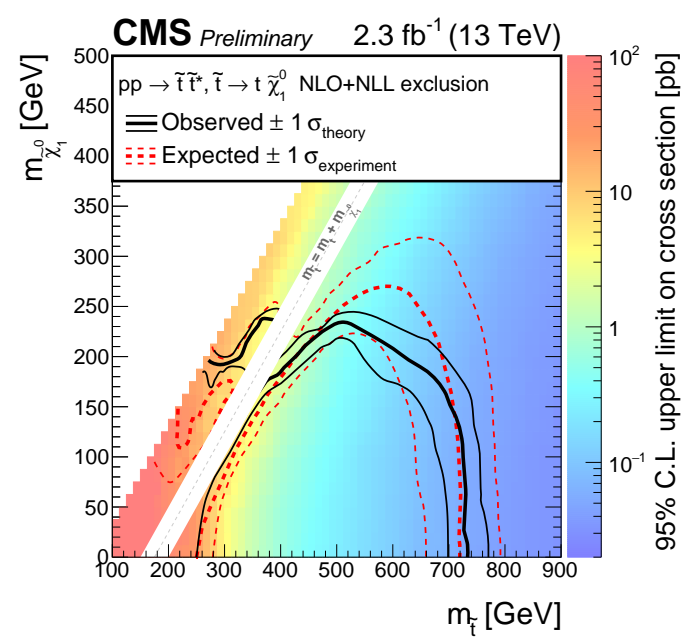

Figure 3: Exclusion limits at $95 \%$ CL for simplified models of top squark pair production obtained by the single lepton channel are shown, assuming that the squark exclusively decay into $\tilde{b_{1}} \rightarrow b \tilde{\chi_{1}^{0}}\left(\tilde{t_{1}} \rightarrow t \tilde{\chi_{1}^{0}}\right)$. The solid black curves represent the observed exclusion contours with respect to NLO+NLL cross section calculations.and the corresponding \pm 1 standard deviations. The dashed red curves indicate the expected exclusion contour and the \pm 1 standard deviations with experimental uncertainties. 

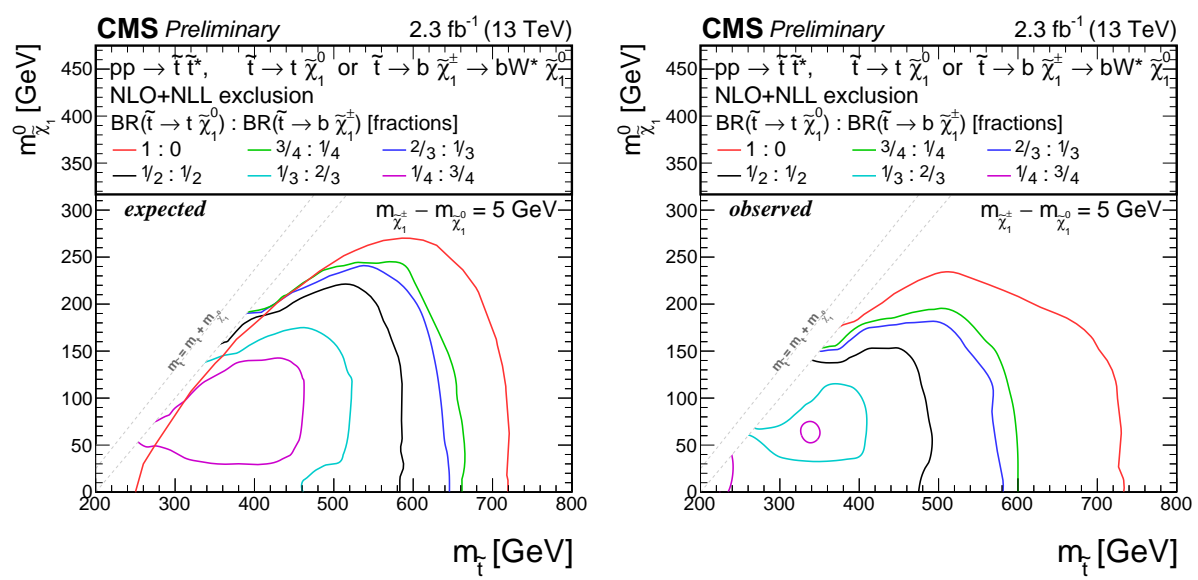

Figure 4: Expected (left) and observed (right) exclusion limits at 95\% CL obtained by the single lepton channel for direct top squark production with different branching fractions for the $\tilde{t_{1}} \rightarrow t^{(*)} \tilde{\chi_{1}^{0}}$ and $\tilde{t_{1}} \rightarrow b \tilde{\chi_{1}^{ \pm}}$ decay modes. The chargino is assumed to be nearly mass-degenerate with the chargino, $m_{\tilde{\chi}_{1}^{ \pm}}=m_{\tilde{\chi}_{1}^{0}}+5 \mathrm{GeV}$, therefore the leptons from the chargino decays are generally too soft to pass the lepton selection.

\section{Sbottom searches}

This section presents a search for direct sbottom pair production based on the presence of bquark jets and large $E_{\mathrm{T}}^{\mathrm{miss}}$. The event selection requires a veto on identified and isolated leptons, a veto on isolated tracks, $E_{\mathrm{T}}^{\text {miss }}>250 \mathrm{GeV}$, exactly two or three jets $p_{\mathrm{T}}>40 \mathrm{GeV}$ and $|\eta|<$ 2.5. Events with a fourth jet with $p_{\mathrm{T}}$ above $50 \mathrm{GeV}$ are rejected. Remaining background mainly originate from top and $\mathrm{W}+$ jets events where a $\mathrm{W}$ decays leptonically but the lepton is not identified. To suppress the background from SM multijet production a minimum difference in azimuthal angle between the $E_{\mathrm{T}}^{\text {miss }}$ vector and each of the leading three jets $\left(\Delta \phi\left(\mathrm{j}_{123}, E_{\mathrm{T}}^{\text {miss }}\right)\right)$ is required to be greater than 0.4 .

For the non-compressed search region targeting signal with a large mass between the sbottom and the neutralino, and additional requirement on the the leading jet $p_{\mathrm{T}}$ to be above $100 \mathrm{GeV}$ and the second jet $p_{\mathrm{T}}$ must to be above $75 \mathrm{GeV}$ are imposed. Both leading jets are required to be $\mathrm{b}-$ tagged. To keep the b-tag efficiency stable as a function of jet $p_{\mathrm{T}}$, as well as to increase the signal to background ratio, two different b-tag working points of the algorithm are used to identify the leading $\mathrm{b}$ jet. A new variable has been introduction, $\min M_{\mathrm{T}}\left(\mathrm{j}, E_{\mathrm{T}}^{\text {miss }}\right)$, defined as a minimal value of $M_{\mathrm{T}}\left(\mathrm{j}, E_{\mathrm{T}}^{\mathrm{miss}}\right)$ computing for the two leading jets:

$$
M_{\mathrm{T}}\left(\mathrm{j}, E_{\mathrm{T}}^{\text {miss }}\right)=\sqrt{2 p_{\mathrm{T}}(j) E_{\mathrm{T}}^{\text {miss }}\left(1+\cos \Delta \phi\left(j, E_{\mathrm{T}}^{\text {miss }}\right)\right)}
$$

This variable is expected to have a kinematic edge at the mass of the top quark when the jet and $E_{\mathrm{T}}^{\text {miss }}$ originate from a semileptonic decay of a top quark. In the non-compressed region the events with $\min M_{\mathrm{T}}\left(\mathrm{j}, E_{\mathrm{T}}^{\text {miss }}\right)$ less than $250 \mathrm{GeV}$ are rejected. To further enhance the signal, the analysis uses the boost-corrected cotransverse mass, $m_{\mathrm{CT}}$, defined as: 


$$
\begin{aligned}
m_{\mathrm{CT}}^{2}\left(j_{1}, j_{2}\right) & =\left[E_{\mathrm{T}}\left(j_{1}\right)+E_{\mathrm{T}}\left(j_{2}\right)\right]^{2}-\left[p_{\mathrm{T}}\left(j_{1}\right)-p_{\mathrm{T}}\left(j_{2}\right)\right]^{2} \\
& =2 p_{\mathrm{T}}\left(j_{1}\right) p_{\mathrm{T}}\left(j_{2}\right)\left(1+\cos \Delta \phi\left(j_{1}, j_{2}\right)\right),
\end{aligned}
$$

For processes with two identical decays of heavy particles, $\tilde{b_{1}} \rightarrow j_{i} \tilde{t}_{1}$ the $m_{\mathrm{CT}}$ distribution is characterized by an endpoint defined by $m\left(\tilde{b_{1}}\right)$ and $m\left(\tilde{\chi}_{1}^{0}\right)$ which for the topology in question is at $\left(m\left(\tilde{b_{1}}\right)^{2}-m\left(\tilde{\chi}_{1}^{0}\right)^{2}\right) / m\left(\tilde{b_{1}}\right)$.

In the compressed region, the decay products have low $p_{\mathrm{T}}$ and are thus hardly visible in the detector in most of the cases. However a hard initial-state-radiation jet (ISR) would boost bottom and top squark products and have more chance to be selected. Therefore a dedicated selection requires the hardest jet to have $p_{\mathrm{T}}>250 \mathrm{GeV}$ and not be b-tagged as the ISR jet is less likely to be originating from a b-quark. The second jet $p_{\mathrm{T}}$ is required to be above $60 \mathrm{GeV}$ and the requirement on $\min M_{\mathrm{T}}\left(\mathrm{j}, E_{\mathrm{T}}^{\text {miss }}\right)$ is relaxed from $250 \mathrm{GeV}$ in the non-compressed region to $200 \mathrm{GeV}$. In addition the leading jet and $E_{\mathrm{T}}^{\text {miss }}$ must to be back-to-back, thus $\Delta \phi\left(\mathrm{j}_{1}, E_{\mathrm{T}}^{\text {miss }}\right)$ is required to be greater than 2.3.

Finally, the events are categorized into 18 exclusive signal regions based on scalar sum of the transverse momenta of the two leading jets $H_{\mathrm{T}}, m_{\mathrm{CT}}$, and $E_{\mathrm{T}}^{\text {miss }}$.

\section{Results and interpretation}

The data are consistent with the background expected from the SM processes further the results are interpreted in term of limits for simplified models. The left plot on Fig 5 shows the expected and observed 95\% CL exclusion limits obtained for a simplified model where the sbottom exclusively decays into $\tilde{b_{1}} \rightarrow b \tilde{\chi_{1}^{0}}$. For small $\tilde{\chi_{1}^{0}}$ masses, the expected limit excludes bottom squark masses below $890 \mathrm{GeV}$ at $95 \% \mathrm{CL}$. The right plot on Fig 5 shows the expected and observed 95\% CL exclusion limits for a simplified model where the top squark exclusively decays into $\tilde{t}_{1} \rightarrow c \tilde{\chi}_{1}^{0}$. Top squarks with masses below $275 \mathrm{GeV}$ are excluded if mass splitting between a top squark and an neutralino is close to $10 \mathrm{GeV}$.

\section{Conclusion}

Four analyses dedicated to the search of direct third generation squark pair production have been presented. The event selection have been optimized for discovery. Several exclusive signal regions have been designed making use of event hypothesis variable and cover a broad range of signal hypothesis from the compressed mass scenario up to the boosted one. Data from the 2015 dataset, corresponding to a luminosity of $2.3 \mathrm{fb}^{-1}$ have been analyzed and no significant excess has been found. Thus the results have been reported in the context of simplified models where an assumption on the mass spectra and the branching ratio is made. The 95\% CL exclusion limits obtained superseed the previous results. Stop masses up to $780 \mathrm{GeV}$ and sbottom masses up to $890 \mathrm{GeV}$ have been probed for low mass neutralino. 

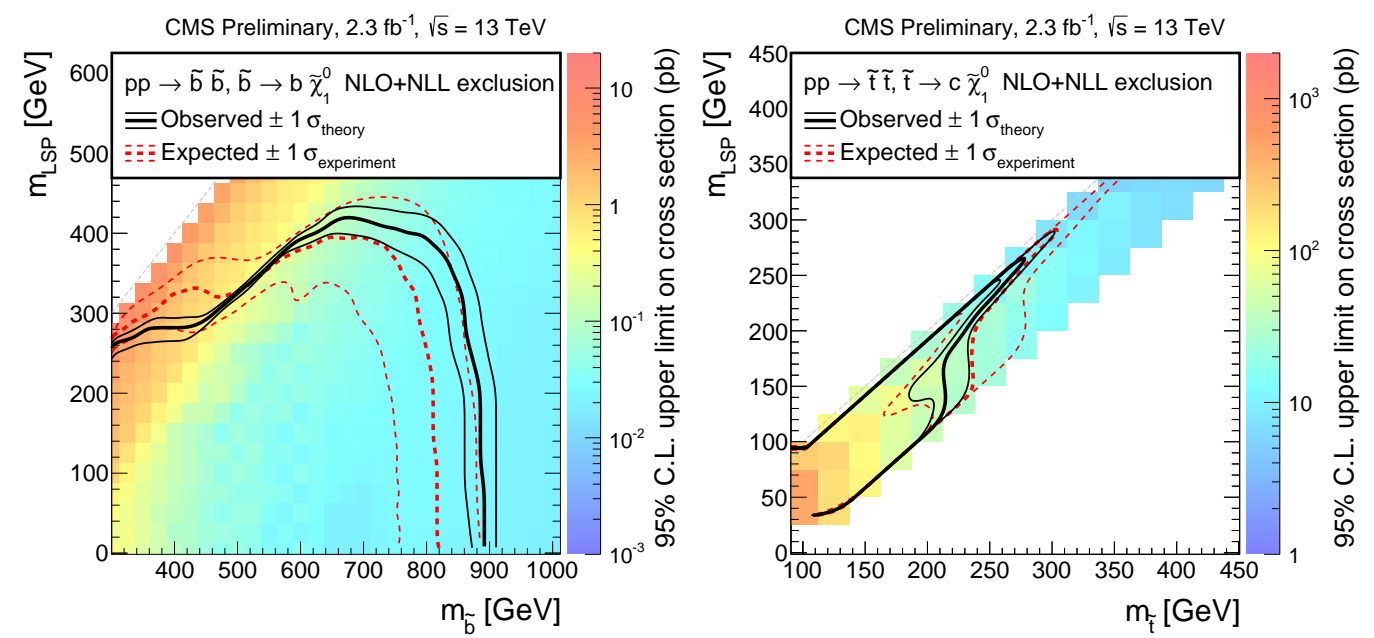

Figure 5: Exclusion limits at 95\% CL for simplified models of bottom (top) squark pair production are shown in left (right). The results on the left (right) assume that the sbottom (squark ) exclusively decay into $\tilde{b_{1}} \rightarrow b \tilde{\chi_{1}^{0}}\left(\tilde{t_{1}} \rightarrow t \tilde{\chi_{1}^{0}}\right)$. The solid black curves represent the observed exclusion contours with respect to NLO+NLL cross section calculations.and the corresponding \pm 1 standard deviations. The dashed red curves indicate the expected exclusion contour and the \pm 1 standard deviations with experimental uncertainties.

\section{References}

[1] S. Chatrchyan et al., "The CMS experiment at the CERN LHC," JINST, vol. 3, p. S08004, 2008.

[2] G. Aad et al., "Search for a supersymmetric partner to the top quark in final states with jets and missing transverse momentum at $\sqrt{s}=7 \mathrm{TeV}$ with the ATLAS detector," Phys. Rev. Lett., vol. 109, p. 211802, 2012.

[3] G. Aad et al., "Search for direct top squark pair production in final states with one isolated lepton, jets, and missing transverse momentum in $\sqrt{s}=7$ tev $p p$ collisions using $4.7 \mathrm{fb}^{-1}$ of atlas data," Phys. Rev. Lett., vol. 109, p. 211803, 2012.

[4] G. Aad et al., "Search for a heavy top-quark partner in final states with two leptons with the atlas detector at the lhc," JHEP, vol. 11, p. 094, 2012.

[5] G. Aad et al., "Search for direct top-squark pair production in final states with two leptons in pp collisions at $\sqrt{s}=8$ tev with the atlas detector," JHEP, vol. 06, p. 124, 2014.

[6] G. Aad et al., "Search for direct third-generation squark pair production in final states with missing transverse momentum and two b-jets in $\sqrt{s}=8 \mathrm{TeV} p p$ collisions with the ATLAS detector," JHEP, vol. 10, p. 189, 2013.

[7] G. Aad et al., "Measurement of spin correlation in top-antitop quark events and search for top squark pair production in pp collisions at $\sqrt{s}=8$ tev using the atlas detector," Phys. Rev. Lett., vol. 114, p. 142001, 2015.

[8] G. Aad et al., "Search for pair-produced third-generation squarks decaying via charm quarks or in compressed supersymmetric scenarios in $p p$ collisions at $\sqrt{s}=8$ tev with the atlas detector," Phys. Rev. D, vol. 90, p. 052008, 2014. 
[9] S. Chatrchyan et al., "Search for top-squark pair production in the single-lepton final state in pp collisions at $\sqrt{s}=8$ tev," Eur. Phys. J. C, vol. 73, p. 2677, 2013.

[10] S. Chatrchyan et al., "Search for supersymmetry in hadronic final states with missing transverse energy using the variables $\alpha_{\mathrm{T}}$ and b-quark multiplicity in pp collisions at $\sqrt{s}=8$ tev," Eur. Phys. J. C, vol. 73 , p. $2568,2013$.

[11] V. Khachatryan et al., "Search for supersymmetry using razor variables in events with $b$-tagged jets in pp collisions at $\sqrt{s}=8$ tev," Phys. Rev. D, vol. 91, p. 052018, 2015.

[12] "Search for direct production of top squark pairs decaying to all-hadronic final states in pp collisions at sqrt(s) = 13 TeV,” Tech. Rep. CMS-PAS-SUS-16-007, CERN, Geneva, 2016.

[13] "Search for direct top squark pair production in the single lepton final state at $\sqrt{s}=13 \mathrm{TeV}$," Tech. Rep. CMS-PAS-SUS-16-002, CERN, Geneva, 2016.

[14] "Search for direct production of bottom and light top squark pairs in proton-proton collisions at $\sqrt{s}=13$ TeV,” Tech. Rep. CMS-PAS-SUS-16-001, CERN, Geneva, 2016. 